\section{Pain Perception in Trout: Not Just Another Fish Story}

Does the catch of the day know that it has been hooked? A new study explores the controversial issue of pain perception in fish, and finds some evidence that fish may feel pain and be able to differentiate between different sources of discomfort.

The concept of animal pain is of considerable concern both to the research community and to society at large, and researchers have explored the extent to which mammals, birds, and even amphibians sense discomfort in the same manner as humans. However, little is known about the extent of nociception in fish.

Pain perception relies on specialized nerve endings near the skin surface that respond to specific noxious or harmful stimuli, such as heat or a poke from a sharp object. Lynne Sneddon and her colleagues at the Roslin Institute (Edinburgh, UK) identified 58 receptors on the face and head of the rainbow trout (Orncorhyncus mykiss), 22 of which could be classified as nociceptors (Proc. R. Soc. Lond. B, 1 June). These receptors were identified through their activation in response to treatment with noxious heat $\left(>40{ }^{\circ} \mathrm{C}\right)$ or chemicals (bee venom or acetic acid).

These stimuli had a profound effect on

Fueyo's group engineered the adenovirus-called Delta-24-RGD—to replicate only in cancer cells by deleting a key amino acid sequence in the viral E1A protein. Healthy cells with functional $\mathrm{Rb}$ proteins are protected against an adenovirus with a disabled E1A protein, but glioma cells with dysfunctional $\mathrm{Rb}$ proteins are unable to prevent Delta-24-RGD from self-replicating, inducing lysis, and infecting other host cancer cells.

When the researchers injected Delta-24RGD into the brain tumors of athymic mice, they observed that 15 of 25 mice $(60 \%)$ survived for more than four months. In contrast, none of the control group mice survived longer than 20 days. Brain examinations of the Delta-24-RGD-treated mice showed complete tumor regression. behavior: fish treated with venom or acid ceased eating for $170 \mathrm{~min}$, nearly three times longer than fish receiving the mock treatment. The former group also exhibited reduced swimming activity and unusual behaviors, such as gently rocking back and forth at the tank bottom or, for acidtreated fish, rubbing their lips against the gravel and tank walls.

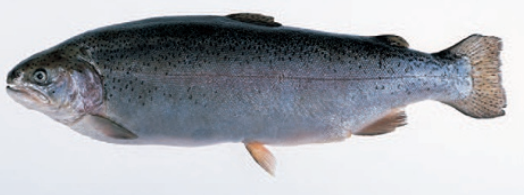

Image courtesy of Regulatory Fish Encyclopedia US Food and Drug Administration, 1993-2003, Photographer, Warren Savary.

Although these findings are far from conclusive as evidence that fish feel pain in the same manner as mammals, they nevertheless indicate that exposure to unpleasant conditions had a lasting impact on the trout. According to the authors, post-treatment behavior seems to mirror actions seen in higher organisms responding to pain, but further investigation will be needed to determine whether these actions are true indicators of pain perception.

$$
-M . E \text {. }
$$

Researchers found empty cavities where the tumors had been and observed no signs of viral gene expression in normal brain tissue.

On the basis of these results, Delta-24RGD is slated for toxicity testing at the National Cancer Institute. "If Delta-24RGD passes the toxicity test, we will focus on the development of a clinical trial [that will involve] glioblastoma patients whose tumors have recurred after being treated with surgery, radiotherapy, and chemotherapy," Fueyo tells Lab Animal. However, he cautions that several obstacles exist in transferring experimental success to human trials. "It might be that the immune system will halt replication of Delta-24-RGD, and by doing so prevent the anticancer effect," he says. 